\title{
Nonconsensual science
}

n May 19, the Institutional Review Board of Loyola University Health System in Chicago approved the institution's involvement in a phase III clinical trial to test the efficacy of the recently developed blood substitute PolyHeme in trauma patients at the scene of injury. This makes Loyola one of twelve level I trauma centers in the country involved in this blood test. Northfield Laboratories Inc., the manufacturer of PolyHeme, hopes the multisite trial will ultimately include $15-20$ centers. Many in emergency medicine have likened the search for a blood substitute to the quest for the Holy Grail, and this trial carries an additional bit of baggage for the expedition; the patients in this clinical trial will be given PolyHeme without prior consent.

Steven Gould, Northfield Laboratories CEO, told the JCI, "The areas that represent the greatest need for rigorous clinical trials occur in emergency, life-threatening situations where there either is no current therapy or the current therapy is inadequate and there is a high mortality [rate]. The central tenant of clinical research is informed consent, but with patients who are in an urgent, life-threatening situation, it is not practicable to obtain informed consent."

To resolve this dilemma, in the mid 1990s, the FDA, the NIH, those in academia and industry, ethicists, and others normally involved in devising new FDA restrictions and regulations came together and drew up a federal regulation that provided a waiver of consent for a select set of clinical trials. This regulation was passed in 1996.

"There are a number of ethicists [who] don't necessarily agree with the regula- tion," Gould noted, but added that for this study, "we are complying with all of the regulatory, legal, and ethical requirements. And we have focused on making certain that every conceivable safeguard is in place to provide maximal assurance of patient safety."

In order for a trial to be considered for a waiver of consent, its protocol must first be approved by the FDA. After that is achieved, the trial must be approved by a local Institutional Review Board (IRB). Obtaining IRB approval requires that an extensive community outreach program be done. This outreach program is meant to alleviate some of the issues raised by the consent waiver; since the patients in such trials cannot speak for themselves, informing the community before the study begins and offering the means to opt out ahead of time by ordering and wearing a bracelet is vital to this process.

"Every site does it differently," Gould told the JCI. "Loyola has done a very good job. They have a very capable crew on this."

At Loyola, Mark Cichon, director of Emergency Medical Services and coinvestigator of the trial, explained that for the process to go smoothly it is essential "to bring everybody to the table initially to get collective feedback from all parties involved - including members of the IRB, the ethicists, the researchers, the clinicians, legal - whatever members are involved in the decision process."

Cichon said the reason he became involved in the trial was that "pre-hospital medicine is my interest. This is something that is a natural extension of the research that

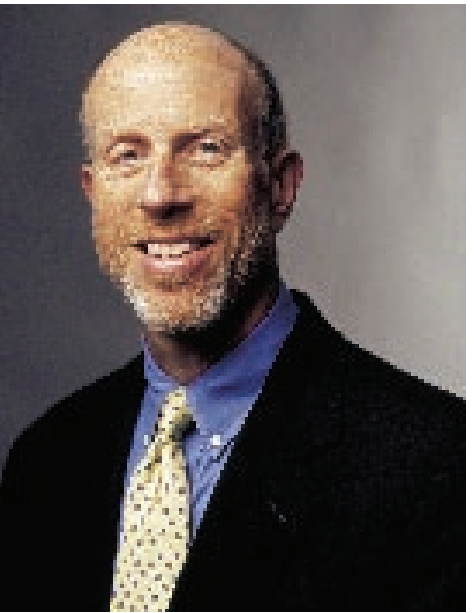

Steven A. Gould has worked since the 1960s to develop an oxygen carrier to be used as a life-saving therapy in situations where blood is not available.

we do with those programs." He believes, however, that "this study is something that could be cutting edge in the sense of making an impact [in an area] that hasn't really changed since the inception of using saline for resuscitation [during] World War I."

Stephen Davidow, head of media relations at Loyola, said that the institution chose the communities based on their location relative to Loyola and to the major expressways that run in and around the Chicago area. "This is a study that is going to take place at the scene of injury and that is primarily going to be [where there have been] car accidents," Davidow explained.

Marcia Halerz, a registered nurse at Loyola and the study coordinator, said

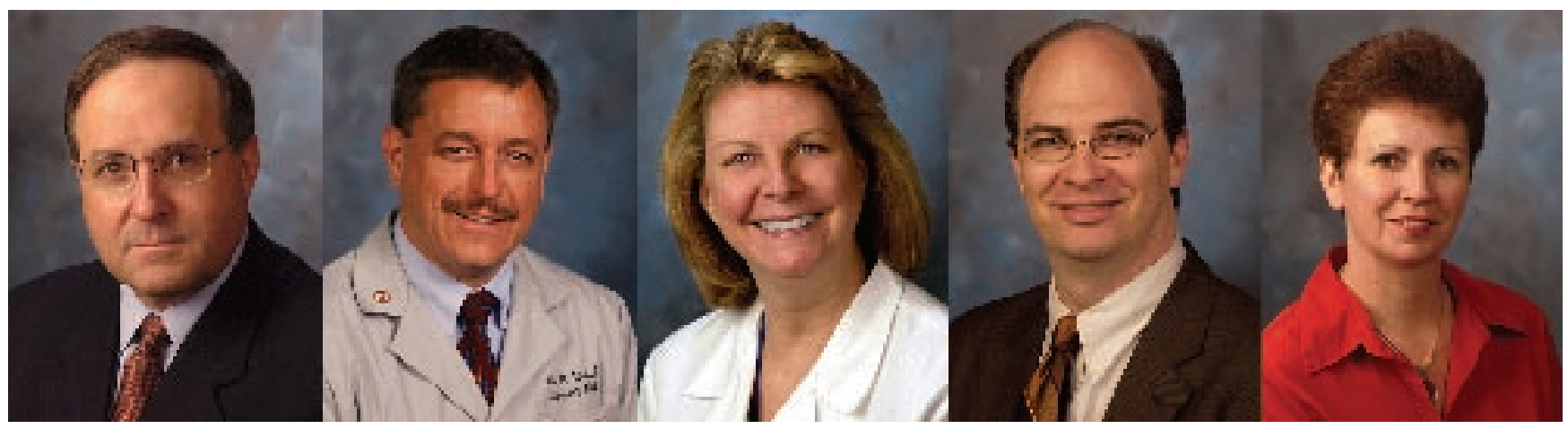

The core outreach group for Loyola University Health System includes (from left to right) principle investigator Richard L. Gamelli, coinvestigator Mark Cinchon, study coordinator Marcia Halerz, head of media relations Stephen Davidow, and outreach program assistant Cathy Monahan. 
that in order to begin the outreach program, "we would go into the community and meet with the local government leaders. That was our first meeting in each area - so that we could inform them about the study, get their permission to go into the towns, and do our community outreach.”

Halerz and Davidow detailed the extensive effort their group made in designing and carrying out plans to reach the people in these communities. Their efforts involved more than 20 specific steps, including creating informational packets; distributing these in schools, churches, community centers, government buildings, public health centers, and other public locations; building an informational website; setting up a phone line and e-mail address for questions and feedback; putting out numerous meeting announcements; and holding two-hour public meetings.

Of those who responded, Davidow said, "Some people, understandably so, were just against the idea of having something that isn't already FDA approved given to them without their consent. Most of the people who came to the meetings were generally quite positive about it. Some were investors so, of course, they were really excited about it."

Halerz, Davidow, and Cichon all noted that anyone planning on doing such a study should be prepared to put in a lot of time and effort.
Yet for all this work, the turnout at the meetings was surprisingly low, averaging, Halerz said, between $0-5$ people. Contact through the phone line and email and responses to the survey were equally limited. Given the extensive and careful nature of Loyola's outreach, it probably cannot be faulted. But with so little response, the question arises: is this in absentia approval for the trial an inherent flaw in the initial regulation whereby people, thinking they personally are unlikely to end up in the trial, don't bother with the outreach and are therefore not truly informed?

\section{Laurie Goodman}

